\title{
Consumption of energy-dense diets in relation to cardiometabolic abnormalities among Iranian women
}

\author{
Ahmad Esmaillzadeh ${ }^{1,2, *}$, Hossein Khosravi Boroujeni ${ }^{1}$ and Leila Azadbakht ${ }^{1,2}$ \\ ${ }^{1}$ Food Security Research Center, Isfahan University of Medical Sciences, Isfahan, Islamic Republic of Iran: \\ ${ }^{2}$ Department of Community Nutrition, School of Nutrition and Food Science, Isfahan University of Medical \\ Sciences, Isfahan, PO Box 81745, Islamic Republic of Iran
}

Submitted 6 April 2011: Accepted 9 September 2011: First published online 19 October 2011

\begin{abstract}
Objective: To assess the association between dietary energy density and the prevalence of cardiometabolic risk factors among Iranian adult women.

Design: Cross-sectional study.

Setting: Tehran, Islamic Republic of Iran.

Subjects: We assessed habitual dietary intakes of 486 Iranian adult women by the use of a validated FFQ. Dietary energy density (DED) was calculated as each individual's reported daily energy intake $(\mathrm{kJ} / \mathrm{d}, \mathrm{kcal} / \mathrm{d})$ divided by the total weight of foods (excluding beverages) consumed ( $\mathrm{g} / \mathrm{d}$ ). Fasting plasma glucose (FPG), lipid profiles and blood pressure were measured. Diabetes (FPG $\geq 126 \mathrm{mg} / \mathrm{dl}$ ), dyslipidaemia (based on the National Cholesterol Education Program Adult Treatment Panel III) and hypertension (based on the Joint National Committee on Prevention, Detection, Evaluation and Treatment of High Blood Pressure) were determined. The presence of 'at least one risk factor' and 'at least two risk factors' of the three major risk factors for CVD (hypertension, dyslipidaemia and diabetes) was also evaluated. To explore the associations between DED and cardiometabolic risk factors, we obtained prevalence ratios in different models accounting for confounders.

Results: Mean DED was $7 \cdot 41$ (SD $1 \cdot 46) \mathrm{kJ} / \mathrm{g}(1 \cdot 77$ (SD 0.35) kcal/g). Consumption of energy-dense diets was associated with higher intakes of energy, dietary fat, cholesterol, vegetable oils, refined grains and high-fat dairy products, along with lower intakes of dietary carbohydrates, fruits, vegetables, and meat and fish. Adherence to an energy-dense diet was associated with elevated levels of serum TAG, total cholesterol and LDL cholesterol and lower levels of serum HDL cholesterol. Women in the top quartile of DED were more likely to have dyslipidaemia $(61 \% v .31 \%, P<0.05)$, at least one $(68 \% v .35 \%, P<0.05)$ and at least two $(29 \% v .10 \%, P<0.05)$ cardiometabolic risk factors compared with those in the bottom quartile. Consumption of energy-dense diets was significantly associated with a greater chance of having dyslipidaemia (prevalence ratio in top quartile $v$. bottom quartile: $1 \cdot 78 ; 95 \%$ CI $1 \cdot 33,2 \cdot 58)$, at least one $(1 \cdot 81 ; 1 \cdot 44,2 \cdot 49)$ and at least two cardiometabolic risk factors $(2 \cdot 81 ; 1 \cdot 51,5 \cdot 24)$. Additional control for BMI and total energy intake slightly attenuated the associations. No overall significant associations were found between consumption of energy-dense diets and risk of having diabetes or hypertension either before or after adjustment for confounders.

Conclusions: Consumption of energy-dense diets was significantly related to the high prevalence of dyslipidaemia, at least one and at least two cardiometabolic risk factors among Iranian adult women. Prospective studies are required to confirm our findings.
\end{abstract}

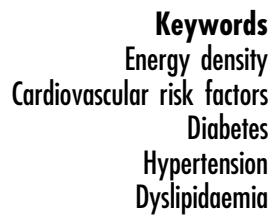

Keywords

factors

ypertension

Dyslipidaemia
The Middle Eastern countries are experiencing a nutrition transition that is shifting from traditional diets high in fibre, fruits and vegetables towards Western diets high in fat, sugar and salt ${ }^{(1)}$. This has resulted in noticeable increases in the prevalence of obesity and its related co-morbidities ${ }^{(2)}$. It has been reported that $25-45 \%$ of total deaths in these countries are due to $\mathrm{CVD}^{(3)}$. Besides being the leading cause of death $^{(4)}$, CVD represents a different picture in this part of the world. Unlike the situation in most developed countries, cardiovascular risk factors are more prevalent among Middle Eastern women than men ${ }^{(5)}$. Furthermore, due to high consumption of refined carbohydrates, low 
HDL cholesterol (HDL-C) and high TAG are the most prevalent cardiovascular risk factors in this part of the world $^{(6)}$. Additionally more than two-thirds of the adult population has dyslipidaemia ${ }^{(7)}$. Furthermore, in contrast to trends observed in northern Europe and the USA, mortality from CVD has risen in the Middle East ${ }^{(8)}$. On the other hand, the majority of available data on dietary determinants of CVD, particularly diet-CVD relationships, has mainly been confined to Western populations with their own dietary characteristics and it is unclear to what extent these findings might apply to the Middle Eastern population taking into account that dietary intakes in this region are totally different from those in Europe, the USA or even other parts of $\mathrm{Asia}^{(9)}$. Therefore, identification of diet-CVD relationships is of great importance in these countries.

Obesity is a major underlying factor contributing to CVD. Recently, great attention has been paid to dietary energy density (DED) in the aetiology of obesity ${ }^{(10-12)}$. DED $(\mathrm{kJ} / \mathrm{g}, \mathrm{kcal} / \mathrm{g})$ has been defined as the amount of energy in a given weight of food ${ }^{(13)}$. It has frequently been reported that consumption of energy-dense diets is significantly associated with greater risk of overweight, obesity or weight gain; not only in cross-sectional ${ }^{(12,14)}$ and prospective studies ${ }^{(15,16)}$, but also in clinical trials, where reducing DED has been associated with significant weight loss ${ }^{(17,18)}$. Obesity has been placed at the centre of studies focusing on DED; however, obesity-related co-morbidities have not received the attention they deserve in this field. Energy-dense diets have been reported to be high in refined grains, processed foods, added sugars and added fats, and low in fruits, vegetables and whole grains - all of these items have been associated with greater risk of CVD. Furthermore, such diets could raise CVD risk through their effects on obesity and weight gain. There are just three reports ${ }^{(19-21)}$ available discussing DED in relation to metabolic risk factors: one has assessed incident diabetes as an outcome ${ }^{(19)}$ among adults in Norfolk, UK; the second ${ }^{(20)}$ found an independent positive association between DED and the metabolic syndrome among US adults; and the third by Japanese investigators failed to find a significant relationship between DED and metabolic risk factors $^{(21)}$. These three studies have their own limitations: the study by Wang et al. ${ }^{(19)}$ just considered diabetes, not other cardiovascular risk factors; Mendoza et $a l^{(20)}$ used a single day's $24 \mathrm{~h}$ dietary recall for collecting dietary data whereas $24 \mathrm{~h}$ dietary recall cannot reflect participants' habitual dietary intakes ${ }^{(22)}$; and Murakami et al.'s ${ }^{(21)}$ study participants were lean young women. To better understand the association between DED and cardiovascular risk factors, we designed the current study in a non-Western population whose dietary intakes have their own unique characteristics ${ }^{(\mathcal{O}, 23)}$. Therefore, the main aim of the present study was to assess the association between DED and cardiovascular risk factors among Iranian women. The second aim was to examine if the associations between DED and metabolic risk factors are mediated through obesity.

\section{Materials and methods}

\section{Participants}

Detailed information about the present cross-sectional study, its participants and measurements has been published earlier ${ }^{(24-26)}$. Briefly, by the use of multistage cluster random sampling method, 583 Tehrani female teachers from different socio-economic categories were invited to participate and 521 women (response rate: 89\%) agreed and gave informed written consent. Exclusion criteria for the current analysis were the existence of a prior history of chronic diseases, leaving more than seventy items blank on the FFQ, under- or over-reporting of energy intake ( $<3347$ or $>17573 \mathrm{~kJ}$ ( $<800$ or $>4200 \mathrm{kcal})$, respectively) and taking medications (propranolol, lovastatin, furosemide, metformin). Therefore, 486 women with full relevant data remained for the current study. The current project was approved by the Research Council of the Food Security and Nutrition Research Center, Isfahan University of Medical Sciences, Isfahan, Iran.

\section{Assessment of dietary intake}

We used a validated 168-item semi-quantitative FFQ for collecting dietary data ${ }^{(27,28)}$. Participants were asked to report the frequency of food items they consumed in the previous year considering a given serving of food items in the questionnaire. They were asked to report on a daily, weekly or monthly basis. We then calculated grams per day of the consumed foods using standard protocols. Total energy intake was calculated by summing up energy intakes from all 168 food items in the questionnaire.

Our validation study ${ }^{(27,28)}$ with the use of biomarkers and also twelve $24 \mathrm{~h}$ dietary recalls as gold standard indicated that the FFQ provided a reasonably valid and reliable measure of intake for most food and nutrient intakes. Although we did not assess the correlation coefficients for DED per se, the correlations for fruits, vegetables and fats (the most influential factors on DED) were reasonable. For instance, comparing two FFQ completed 1 year apart, we reached the correlation coefficients of 0.71 for reproducibility of fruits, 0.74 for vegetables, 0.59 for partially hydrogenated and 0.69 for non-hydrogenated vegetable oils. The validity of the FFQ for assessing consumption of fruits, vegetables, partially hydrogenated and non-hydrogenated vegetable oils was also good, such that between the FFQ and detailed dietary recalls, corresponding correlation coefficients were $0 \cdot 61,0.57,0.55$ and $0 \cdot 44$, respectively.

\section{Calculation of dietary energy density}

To calculate DED, we divided each individual's reported daily energy intake from foods only $(\mathrm{kJ} / \mathrm{d}, \mathrm{kcal} / \mathrm{d})$ into total weight of foods consumed $(\mathrm{g} / \mathrm{d})$. Total weight of foods consumed by participants was calculated by summing up the weight of foods only (154 out of 168 food items in the FFQ). We did not consider energy and weight of drinks consumed because a recent systematic review 
has shown that calculating DED by including drinks results in a variable that biases associations between DED and weight gain towards the null ${ }^{(29)}$. Earlier studies have shown that calculating DED based on foods only would provide a variable with the best associations with obesity and weight gain ${ }^{(30)}$.

\section{Assessment of other variables}

Anthropometric variables including weight, height and waist circumference were measured ${ }^{(9)}$ and BMI was calculated as weight in kilograms divided by the square of height in metres. Plasma glucose concentrations and serum lipid levels were measured on a fasting blood sample by the use of a Selectra 2 auto-analyser (Vital Scientific, Spankeren, Netherlands) ${ }^{(28)}$. Fasting plasma glucose (FPG) was measured on the day of blood collection by the enzymatic colorimetric method using glucose oxidase (Pars Azmoon Inc., Tehran, Iran). Serum TAG concentrations were assayed using TAG kits (Pars Azmoon Inc.) by enzymatic colorimetric tests with glycerol phosphate oxidase. Serum HDL-C was measured after precipitation of the apo-B-containing lipoproteins with phosphotungstic acid. Serum LDL cholesterol (LDL-C) was calculated from serum total cholesterol (TC), TAG and HDL-C, except when TAG concentration was greater than $4.4 \mathrm{mmol} / \mathrm{l}(400 \mathrm{mg} / \mathrm{dl})^{(31)}$. The inter- and intra-assay CV of this method were $<10 \%$. Blood pressure was measured three times after the participants sat for $15 \mathrm{~min}$, as reported earlier $^{(26)}$. We used the International Physical Activity Questionnaire for obtaining data on physical activity ${ }^{(32)}$. Using standard guidelines ${ }^{(33)}$, these data were expressed as metabolic equivalent hours per week (MET-h/week). Additional information regarding age, smoking habits, socio-economic status (defined based on educational level, income, family size, being owner of the house or renting the house, house area), medical history and current use of medications was obtained using questionnaires.

\section{Definition of terms}

Hypertriacylglycerolaemia was defined as serum TAG $\geq 2 \cdot 2 \mathrm{mmol} / \mathrm{l} \quad(200 \mathrm{mg} / \mathrm{dl})$, hypercholesterolaemia as serum TC $\geq 6 \cdot 24 \mathrm{mmol} / 1(240 \mathrm{mg} / \mathrm{dl})$, high serum LDL-C as $\geq 4 \cdot 1 \mathrm{mmol} / 1(160 \mathrm{mg} / \mathrm{dl})$ and low serum HDL-C as $<1.29 \mathrm{mmol} / \mathrm{l}(50 \mathrm{mg} / \mathrm{dl})^{(35)}$. Dyslipidaemia was defined based on the third report of the National Cholesterol Education Program Adult Treatment Panel III ${ }^{(34)}$ as having hypertriacylglycerolaemia or hypercholesterolaemia or high LDL-C or low HDL-C. Hypertension was defined as systolic blood pressure $\geq 140 \mathrm{mmHg}$ or diastolic blood pressure $\geq 90 \mathrm{mmHg}$ based on the sixth report of the Joint National Committee on Prevention, Detection, Evaluation and Treatment of High Blood Pressure ${ }^{(35)}$. Diabetes mellitus was defined as FPG $\geq 6.93 \mathrm{mmol} / \mathrm{l}^{(36)}$. The presence of 'at least one risk factor' and 'at least two risk factors' of the three major risk factors for CVD (hypertension, dyslipidaemia and diabetes) was also evaluated.

\section{Statistical methods}

Quartile cut-off points of DED were used for categorizing participants. General characteristics and dietary intakes of participants were compared across quartiles of DED by the use of ANOVA, $\chi^{2}$ and analysis of covariance (ANCOVA) where appropriate. All dietary intakes were obtained by the use of General Linear Models with age and total energy intake as covariates. Multivariateadjusted means for metabolic risk factors were compared across quartiles of DED by the use of ANCOVA. Selection of variables to be included in the models was done using the change-in-estimate method. This method has been shown to produce more reliable models than the methods based on statistical significance ${ }^{(37)}$. Therefore, a potential confounder is included in the model if it changed the coefficient by $10 \%$. This approach is consistent with the definition of confounding, where confounding is said to be present if the unadjusted effect differs from the effect adjusted for putative confounders ${ }^{(37)}$. Adjustments were made for age, cigarette smoking, physical activity, socio-economic status, current oestrogen use, menopausal status, family history of diabetes and stroke, and BMI. To explore the associations between DED and cardiometabolic risk factors, we obtained prevalence ratios in different models. First, we adjusted for age (years), cigarette smoking (yes or no), physical activity (MET-h/week), socio-economic status (categorical), current oestrogen use (yes or no), menopausal status (yes or no) and family history of diabetes and stroke (yes or no). Then adjustments were made for BMI. All models were done by treating DED as quartile categories with the first quartile as a reference. The overall trend across quartiles of DED was examined through the use of the Mantel-Haenszel extension $\chi^{2}$ test. The SPSS statistical software package version $10 \cdot 0$ (SPSS Inc., Chicago, IL, USA) was used for all statistical analyses. $P \leq 0.05$ was taken to indicate statistical significance.

\section{Results}

Mean DED was 7•41 (sD 1.46) kJ/g (1.77 (sD 0.35) kcal/g). General characteristics and dietary intakes of women across quartiles of DED are shown in Table 1. Individuals in the top quartile of DED were younger, less physically active, less likely to be postmenopausal and had higher BMI compared with those in the lowest quartile. Consumption of energy-dense diets was associated with higher intakes of energy, dietary fat, cholesterol, vegetable oils, refined grains and high-fat dairy products, along with lower intakes of dietary carbohydrates, fruits, vegetables, and meat and fish.

Multivariate-adjusted means for metabolic risk factors across quartiles of DED are provided in Table 2. After control for potential confounders, participants in the highest quartile of energy density had elevated levels of 
Table 1 General characteristics and dietary intakes of 486 Tehrani females by quartile of dietary energy density (DED)*

\begin{tabular}{|c|c|c|c|c|c|c|c|c|c|}
\hline & \multicolumn{8}{|c|}{ Quartile of DED } & \multirow[b]{3}{*}{$P$ for trendt } \\
\hline & \multicolumn{2}{|c|}{1 (lowest) } & \multicolumn{2}{|c|}{2} & \multicolumn{2}{|c|}{3} & \multicolumn{2}{|c|}{4 (highest) } & \\
\hline & Mean & SD & Mean & SD & Mean & SD & Mean & SD & \\
\hline$n$ & \multicolumn{2}{|c|}{122} & \multicolumn{2}{|c|}{121} & \multicolumn{2}{|c|}{121} & \multicolumn{2}{|c|}{122} & - \\
\hline Range $(\mathrm{kJ} / \mathrm{g})$ & \multirow{2}{*}{\multicolumn{2}{|c|}{$\begin{array}{l}<6.28 \\
<1.50\end{array}$}} & \multirow{2}{*}{\multicolumn{2}{|c|}{$\begin{array}{l}6.28-<7.66 \\
1.50-<1.83\end{array}$}} & \multirow{2}{*}{\multicolumn{2}{|c|}{$\begin{array}{l}7.66-<8.58 \\
1.83-<2.05\end{array}$}} & \multicolumn{2}{|c|}{$\geq 8.58$} & - \\
\hline Range $(\mathrm{kcal} / \mathrm{g})$ & & & & & & & \multicolumn{2}{|c|}{$\geq 2 \cdot 05$} & - \\
\hline Age (year) & 54 & 5 & \multicolumn{2}{|c|}{$49 \quad 6$} & \multicolumn{2}{|c|}{$50 \quad 6$} & 44 & 6 & $<0.01$ \\
\hline BMl $\left(\mathrm{kg} / \mathrm{m}^{2}\right)$ & $25 \cdot 9$ & 3.5 & $26 \cdot 9$ & $3 \cdot 8$ & 28 . & $3 \cdot 3$ & 28 & $3 \cdot 7$ & $<0.01$ \\
\hline Physical activity (MET-h/week) & $16 \cdot 3$ & $10 \cdot 9$ & $16 \cdot 1$ & $11 \cdot 2$ & 14 & $9 \cdot 9$ & 11 & $10 \cdot 1$ & $<0.01$ \\
\hline Family history of diabetes (\%) & \multicolumn{2}{|c|}{9} & \multicolumn{2}{|c|}{9} & \multicolumn{2}{|c|}{8} & \multicolumn{2}{|c|}{6} & 0.07 \\
\hline Family history of stroke (\%) & \multicolumn{2}{|c|}{1} & \multicolumn{2}{|c|}{0} & \multicolumn{2}{|c|}{1} & \multicolumn{2}{|c|}{1} & 0.59 \\
\hline Current daily smoker (\%) & \multirow{2}{*}{\multicolumn{2}{|c|}{$\begin{array}{r}2 \\
24\end{array}$}} & \multirow{2}{*}{\multicolumn{2}{|c|}{$\begin{array}{r}1 \\
28\end{array}$}} & & & & & $0 \cdot 71$ \\
\hline Obese $(\%) \ddagger$ & & & & & & & & & $<0.01$ \\
\hline Current oestrogen use (\%) & & & 2 & & & & & & $0 \cdot 11$ \\
\hline Postmenopausal (\%) & & & 5 & & & & & & $<0.01$ \\
\hline Dietary intakes: nutrients & & & & & & & & & \\
\hline Total energy $(\mathrm{kJ} / \mathrm{d})$ & 9719 & 109 & 10058 & 100 & 10381 & 100 & 10849 & 113 & $<0.05$ \\
\hline Total energy $(\mathrm{kcal} / \mathrm{d})$ & 2323 & 26 & 2404 & 24 & 2481 & 24 & 2593 & 27 & $<0.05$ \\
\hline Carbohydrate (\%E) & 61 & 1 & 61 & 1 & 58 & 1 & 57 & 1 & $<0.05$ \\
\hline Protein (\%E) & 14 & 0.7 & 13 & 0.6 & 14 & $0 \cdot 7$ & 12 & 0.6 & $0 \cdot 68$ \\
\hline Fat $(\% \mathrm{E})$ & 25 & 0.6 & 26 & 0.8 & 28 & 0.7 & 31 & $0 \cdot 7$ & $<0.05$ \\
\hline Cholesterol (mg/d) & 154 & 10 & 149 & 9 & 191 & 11 & 206 & 8 & $<0.01$ \\
\hline Dietary fibre $(\mathrm{g} / \mathrm{d})$ & 18 & 1 & 17 & 1 & 15 & 1 & 13 & 1 & 0.09 \\
\hline Dietary intakes: food groups $(\mathrm{g} / \mathrm{d})$ & & & & & & & & & \\
\hline Partially hydrogenated vegetable oils & 16 & 1 & 18 & 1 & 24 & 1 & 32 & 1 & $<0.01$ \\
\hline Non-hydrogenated vegetable oils & 21 & 1 & 18 & 1 & 20 & 1 & 29 & 1 & $<0.05$ \\
\hline Fruits & 257 & 9 & 249 & 10 & 221 & 8 & 179 & 9 & $<0.01$ \\
\hline Vegetables & 245 & 8 & 217 & 7 & 196 & 5 & 169 & 7 & $<0.01$ \\
\hline Meat and fish & 98 & 3 & 94 & 4 & 89 & 3 & 81 & 3 & $<0.05$ \\
\hline Whole grains & 121 & 4 & 119 & 4 & 115 & 3 & 112 & 4 & 0.33 \\
\hline Refined grains & 179 & 7 & 201 & 8 & 213 & 7 & 220 & 8 & $<0.05$ \\
\hline Low-fat dairy & 96 & 3 & 95 & 4 & 97 & 4 & 90 & 4 & 0.51 \\
\hline High-fat dairy & 82 & 3 & 87 & 3 & 96 & 3 & 117 & 5 & $<0.05$ \\
\hline
\end{tabular}

$\% \mathrm{E}$, percentage of total energy intake.

*Data are means and standard deviations unless indicated otherwise.

tBy using linear regression.

$\neq$ Obesity: BMI $\geq 30 \mathrm{~kg} / \mathrm{m}^{2}$.

serum TAG, TC and LDL-C and lower levels of serum HDL-C compared with those in the lowest quartile. Higher DED was not associated with FPG levels, systolic and diastolic blood pressure.

The prevalences of cardiometabolic risk factors across quartiles of DED are shown in Fig. 1. Women in the top quartile of DED were more likely to have dyslipidaemia, at least one and at least two cardiometabolic risk factors. No overall significant association was found between DED and prevalence of diabetes and hypertension.

Multivariate-adjusted prevalence ratios for cardiometabolic abnormalities across quartiles of DED are presented in Table 3. After controlling for age and other potential confounders, consumption of energy-dense diets was significantly associated with greater chance of having dyslipidaemia (prevalence ratio in top quartile $v$. bottom quartile: $1 \cdot 78 ; 95 \%$ CI $1 \cdot 33,2 \cdot 58)$, at least one $(1 \cdot 81 ; 1 \cdot 44$, $2 \cdot 49)$ and at least two cardiometabolic risk factors $(2 \cdot 81$; $1 \cdot 51,5 \cdot 24)$. Additional control for BMI slightly attenuated the associations but all were still significant. Even after further adjustment for total energy intake the associations remained significant. No overall significant associations were found between consumption of energy-dense diets and risk of having diabetes or hypertension either before or after adjustment for confounders.

\section{Discussion}

We found that consumption of energy-dense diets was associated with higher risk of having dyslipidaemia, at least one and at least two cardiometabolic risk factors. No overall significant associations were found between DED and the prevalence of hypertension and diabetes. To the best of our knowledge, the present study is among the first to examine DED in relation to cardiometabolic abnormalities in a Middle Eastern population.

Several controversial methods have been proposed for calculating DED. Some investigators have suggested the use of drinks in DED calculation, others disagree ${ }^{(38,39)}$. In a recent systematic review ${ }^{(29)}$, it was suggested that drinks must be excluded from the calculation of DED and including 'foods only' would better reflect the meaning of DED. This conclusion was based on findings from crosssectional or prospective studies indicating the effect of DED on body weight ${ }^{(14,20,40)}$. In the current study, only 
Table 2 Multivariate-adjusted means of cardiovascular risk factors across quartiles of dietary energy density (DED) among 486 Tehrani females*

\begin{tabular}{|c|c|c|c|c|c|c|c|c|c|}
\hline & \multicolumn{8}{|c|}{ Quartile of DED } & \multirow[b]{3}{*}{$P$ for trend } \\
\hline & \multicolumn{2}{|c|}{1 (lowest) } & \multicolumn{2}{|c|}{2} & \multicolumn{2}{|c|}{3} & \multicolumn{2}{|c|}{4 (highest) } & \\
\hline & Mean & SE & Mean & SE & Mean & SE & Mean & SE & \\
\hline$n$ & \multicolumn{2}{|c|}{122} & \multicolumn{2}{|c|}{121} & \multicolumn{2}{|c|}{121} & \multicolumn{2}{|c|}{122} & - \\
\hline Range (kJ/g) & \multirow{2}{*}{\multicolumn{2}{|c|}{$\begin{array}{l}<6.28 \\
<1.50\end{array}$}} & \multirow{2}{*}{\multicolumn{2}{|c|}{$\begin{array}{l}6 \cdot 28-<7 \cdot 66 \\
1 \cdot 50-<1 \cdot 83\end{array}$}} & \multirow{2}{*}{\multicolumn{2}{|c|}{$\begin{array}{l}7.66-<8.58 \\
1.83-<0.05\end{array}$}} & \multirow{2}{*}{\multicolumn{2}{|c|}{$\begin{array}{l}\geq 8.58 \\
\geq 2.05\end{array}$}} & - \\
\hline Range $(\mathrm{kcal} / \mathrm{g})$ & & & & & & & & & - \\
\hline $\mathrm{FPG}(\mathrm{mg} / \mathrm{dl})$ & 95 & 2 & 97 & 2 & 96 & 2 & 98 & 2 & 0.23 \\
\hline Serum TAG $(\mathrm{mg} / \mathrm{dl})$ & 149 & 8 & 167 & 7 & 180 & 7 & 196 & 9 & $<0.01$ \\
\hline Serum TC $(\mathrm{mg} / \mathrm{dl})$ & 186 & 3 & 194 & 3 & 207 & 2 & 229 & 2 & $<0.05$ \\
\hline Serum HDL-C (mg/dl) & 55 & 1 & 48 & 1 & 39 & 1 & 34 & 1 & $<0.01$ \\
\hline Serum LDL-C (mg/dl) & 131 & 2 & 127 & 3 & 142 & 2 & 149 & 2 & $<0.05$ \\
\hline $\mathrm{SBP}(\mathrm{mmHg})$ & 124 & 1 & 126 & 2 & 123 & 1 & 127 & 1 & 0.29 \\
\hline $\mathrm{DBP}(\mathrm{mmHg})$ & 83 & 1 & 85 & 1 & 85 & 1 & 84 & 1 & 0.51 \\
\hline
\end{tabular}

FPG, fasting plasma glucose; TC, total cholesterol; HDL-C, HDL cholesterol; LDL-C, LDL cholesterol; SBP, systolic blood pressure; DBP diastolic blood pressure.

${ }^{*}$ Data are means with their standard errors adjusted for age, cigarette smoking, physical activity, socio-economic status, current oestrogen use, menopausal status, family history of diabetes and stroke, and BMI.

tBy using linear regression.

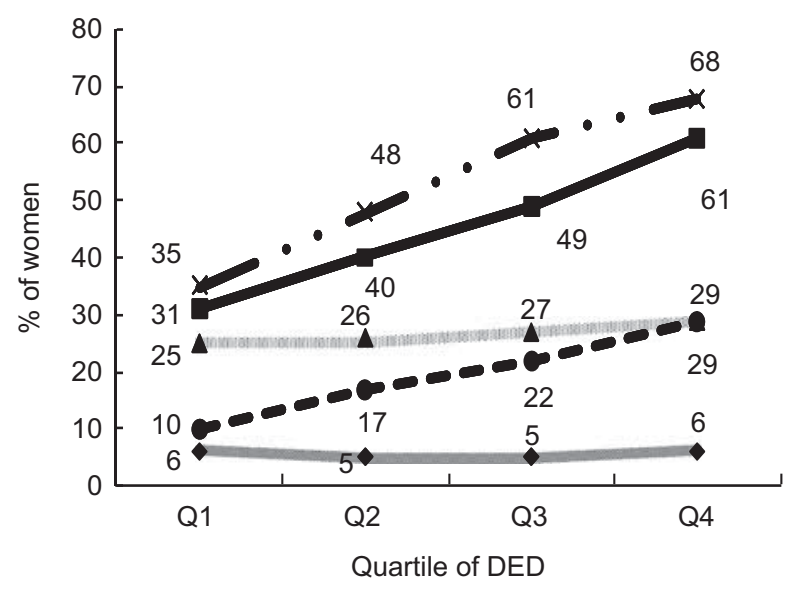

Fig. 1 Prevalence of cardiovascular risk factors ( diabetes; - $\longrightarrow$, dyslipidaemia; $\boldsymbol{\Lambda}$, hypertension; $-\times-$, at least one risk factor (ALORF); - - - , at least two risk factors (ALTRF)) across quartiles of dietary energy density (DED) among 486 Tehrani females. Diabetes was defined as fasting plasma glucose $\geq 126 \mathrm{mg} / \mathrm{dl}$. Dyslipidaemia was defined as having hypertriacylglycerolaemia (serum TAG $\geq 200 \mathrm{mg} / \mathrm{dl}$ ) or hypercholesterolaemia (serum total cholesterol $\geq 240 \mathrm{mg} / \mathrm{dl}$ ) or high LDL cholesterol ( $\geq 160 \mathrm{mg} / \mathrm{dl}$ ) or low HDL cholesterol $(<50 \mathrm{mg} / \mathrm{dl})$. Hypertension was defined as systolic blood pressure $\geq 140 \mathrm{mmHg}$ or diastolic blood pressure $\geq 90 \mathrm{mmHg}$ based on sixth report of the Joint National Committee on Prevention, Detection, Evaluation and Treatment of High Blood Pressure. ALORF and ALTRF were defined as having at least one and two cardiometabolic risk factors, respectively, from among the three major risk factors of hypertension, dyslipidaemia and diabetes. Women in the top quartile of DED were more likely to have dyslipidaemia, at least one and at least two cardiometabolic risk factors

foods, not drinks, were considered for calculating DED. Such method of calculation would not bias the associations towards the null ${ }^{(29)}$. However, when we considered beverages in the calculation of DED, the associations were slightly attenuated but their significance did not alter. Mean
DED obtained in the present study is slightly higher than that in other investigations using the same method.

We found that high DED is associated with unhealthy food choices. This finding is in agreement with other studies where DED has been related to poor diet quality $^{(17,41)}$. Energy-dense diets are often high in refined grains, processed foods, added sugars and added fats, and low in fruits, vegetables and whole grains. The main characteristic of energy-dense diets, as shown by BesRastrollo et $a l^{(16)}$, is that they contain higher amounts of saturated and trans fats and a higher glycaemic load. It must be kept in mind that high-fat diets do not necessarily contain higher energy density. A prominent example in this case is the traditional Mediterranean dietary pattern. Despite the higher content of fat, this dietary pattern is also popular for its higher content of fruits and vegetables. In Japan, low-energy-dense diets have been reported to be high in boiled rice and noodles ${ }^{(21)}$. In the 1994-1996 Continuing Survey of Food Intakes by Individuals, Ledikwe et al. ${ }^{(42)}$ have shown that consumption of a low-energy-dense diet is associated with higher intakes of micronutrient-rich foods high in water and low in fat, lower intakes of total dietary fat and carbonated beverages, and higher intakes of vitamins $\mathrm{A}, \mathrm{C}$ and $\mathrm{B}_{6}$, folate, $\mathrm{Fe}$, $\mathrm{Ca}$ and $\mathrm{K}$. It seems that low-energy-dense diets in different parts of the world have their own characteristics: in some Asian populations they contain foods high in water, whereas in Western populations they contain a lower content of fat. The diet in Middle Eastern countries is totally different from that in the USA, Europe and also other parts of Asia. However, it seems that the characteristics of energy-dense diets in Iran are similar to those in Western populations. Characteristics of energydense diets are particularly important when reducing DED is chosen as a strategy for weight management. What is the best way to reach reduced DED is still under investigation. Some have suggested reduced intakes of 
Table 3 Multivariate-adjusted prevalence ratios and 95\% confidence intervals for cardiovascular risk factors across quartiles of dietary energy density (DED) among 486 Tehrani females ${ }^{\star}+$

\begin{tabular}{|c|c|c|c|c|c|c|c|c|}
\hline & \multicolumn{7}{|c|}{ Quartile of DED } & \multirow[b]{3}{*}{$P$ for trend $\ddagger$} \\
\hline & \multirow[b]{2}{*}{1 (lowest) } & \multicolumn{2}{|c|}{2} & \multicolumn{2}{|c|}{3} & \multicolumn{2}{|c|}{4 (highest) } & \\
\hline & & PR & $95 \% \mathrm{Cl}$ & PR & $95 \% \mathrm{Cl}$ & PR & $95 \% \mathrm{Cl}$ & \\
\hline$n$ & 122 & \multicolumn{2}{|c|}{121} & \multicolumn{2}{|c|}{121} & \multicolumn{2}{|c|}{122} & - \\
\hline Range $(\mathrm{kJ} / \mathrm{g})$ & $<6.28$ & \multicolumn{2}{|c|}{$6 \cdot 28-<7 \cdot 66$} & \multirow{2}{*}{\multicolumn{2}{|c|}{$7 \cdot 66-<8.58$}} & \multicolumn{2}{|c|}{$\geq 8.58$} & - \\
\hline Range $(\mathrm{kcal} / \mathrm{g})$ & $<1.50$ & \multicolumn{2}{|c|}{$1 \cdot 50-<1.83$} & & $1 \cdot 83-<2 \cdot 05$ & \multicolumn{2}{|c|}{$\geq 2.05$} & - \\
\hline \multicolumn{9}{|l|}{ Diabetes } \\
\hline Crude & $1 \cdot 00$ & 0.83 & $0.29,6.64$ & 0.83 & $0.29,6.64$ & 1.00 & $0.36,2 \cdot 76$ & 0.39 \\
\hline Model I & 1.00 & 0.88 & $0.27,6.61$ & 0.85 & $0.29,6.58$ & $1 \cdot 02$ & $0 \cdot 38,2 \cdot 71$ & 0.31 \\
\hline Model II & 1.00 & 0.90 & $0.28,6.55$ & 0.88 & $0.30,6 \cdot 61$ & 1.06 & $0.42,2.73$ & 0.29 \\
\hline \multicolumn{9}{|l|}{ Dyslipidaemia } \\
\hline Crude & $1 \cdot 00$ & $1 \cdot 29$ & $1 \cdot 21,1 \cdot 37$ & 1.58 & $1 \cdot 15,2 \cdot 18$ & 1.97 & $1 \cdot 46,2 \cdot 66$ & $<0.01$ \\
\hline Model I & 1.00 & $1 \cdot 13$ & $1 \cdot 06,1 \cdot 30$ & $1 \cdot 40$ & $1 \cdot 13,2 \cdot 11$ & $1 \cdot 78$ & $1 \cdot 33,2 \cdot 58$ & $<0.01$ \\
\hline Model II & $1 \cdot 00$ & $1 \cdot 04$ & $0.92,1.19$ & $1 \cdot 28$ & $1 \cdot 06,2 \cdot 01$ & 1.64 & $1 \cdot 21,2 \cdot 56$ & $<0.01$ \\
\hline \multicolumn{9}{|l|}{ Hypertension } \\
\hline Crude & $1 \cdot 00$ & $1 \cdot 04$ & $0.67,1.60$ & 1.08 & $0.70,1.65$ & $1 \cdot 16$ & $0.76,1 \cdot 76$ & 0.23 \\
\hline Model I & $1 \cdot 00$ & $1 \cdot 02$ & $0.65,1.57$ & 1.07 & $0 \cdot 73,1 \cdot 61$ & $1 \cdot 13$ & $0.73,1 \cdot 71$ & $0 \cdot 21$ \\
\hline Model II & $1 \cdot 00$ & 0.96 & $0.61,1.51$ & 1.04 & $0.69,1.59$ & $1 \cdot 10$ & $0.79,1.75$ & $0 \cdot 15$ \\
\hline \multicolumn{9}{|l|}{ ALORF } \\
\hline Crude & $1 \cdot 00$ & $1 \cdot 37$ & $1 \cdot 01,1 \cdot 86$ & $1 \cdot 74$ & $1 \cdot 32,2 \cdot 30$ & 1.94 & $1 \cdot 48,2 \cdot 54$ & $<0.01$ \\
\hline Model I & 1.00 & $1 \cdot 19$ & $0.95,1.79$ & 1.56 & $1 \cdot 25,2 \cdot 19$ & $1 \cdot 81$ & $1 \cdot 44,2 \cdot 49$ & $<0.01$ \\
\hline Model II & 1.00 & $1 \cdot 11$ & $0.89,1.83$ & $1 \cdot 40$ & $1 \cdot 17,2 \cdot 08$ & 1.68 & $1 \cdot 36,2 \cdot 38$ & $<0.01$ \\
\hline \multicolumn{9}{|l|}{ ALTRF } \\
\hline Crude & $1 \cdot 00$ & $1 \cdot 70$ & $0.87,3.30$ & $2 \cdot 20$ & $1 \cdot 69,4 \cdot 13$ & $2 \cdot 90$ & $1 \cdot 58,5 \cdot 31$ & $<0.001$ \\
\hline Model I & 1.00 & $1 \cdot 54$ & $0.81,3.19$ & 1.98 & $1.63,3.93$ & $2 \cdot 81$ & $1 \cdot 51,5 \cdot 24$ & $<0.001$ \\
\hline Model II & 1.00 & $1 \cdot 43$ & $0.78,3.11$ & $1 \cdot 87$ & $1 \cdot 65,3 \cdot 84$ & $2 \cdot 69$ & $1 \cdot 40,5 \cdot 13$ & $<0.001$ \\
\hline
\end{tabular}

PR, prevalence ratio; ALORF, at least one risk factor; ALTRF, at least two risk factors.

*Diabetes was defined as fasting plasma glucose $\geq 126 \mathrm{mg} / \mathrm{dl}$. Dyslipidaemia was defined as having hypertriacylglycerolaemia (serum TAG $\geq 200 \mathrm{mg} / \mathrm{dl}$ ) or hypercholesterolaemia (serum total cholesterol $\geq 240 \mathrm{mg} / \mathrm{dl}$ ) or high LDL cholesterol ( $\geq 160 \mathrm{mg} / \mathrm{dl}$ ) or low HDL cholesterol ( $<50 \mathrm{mg} / \mathrm{dl}$ ). Hypertension was defined as systolic blood pressure $\geq 140 \mathrm{mmHg}$ or diastolic blood pressure $\geq 90 \mathrm{mmHg}$ based on sixth report of the Joint National Committee on Prevention, Detection, Evaluation and Treatment of High Blood Pressure. ALORF and ALTRF were defined as having at least one and two cardiometabolic risk factors, respectively, from among three major risk factors of hypertension, dyslipidaemia and diabetes.

tModel I: adjusted for age, cigarette smoking, physical activity, socio-economic status, current oestrogen use, menopausal status and family history of diabetes and stroke. Model II: additionally adjusted for BMI.

$\ddagger$ By the use of Mantel-Haenszel extension $\chi^{2}$ test.

foods rich in saturated fats and refined carbohydrates and a simultaneous increase in fruit and vegetable intakes ${ }^{(16)}$. Others believe that reducing the consumption of soft drinks is the case ${ }^{(41)}$.

We found a significant positive association between DED and the prevalence of dyslipidaemia. However no significant association was found with DED, hypertension and diabetes. Although previous studies have found a positive association between DED and the metabolic syndrome $^{(20)}$, no one has reported such an association with dyslipidaemia. In a cross-sectional study among young Japanese women aged 18-22 years, Murakami et al. ${ }^{(21)}$ failed to find a significant relationship between DED and metabolic variables such as plasma glucose levels, blood lipid profiles and blood pressure. The difference between our findings and those of Murakami et al. ${ }^{(21)}$ might be explained by the characteristics of the study population. In the Japanese study lean young women were studied, while ours was an overweight population. In contrast to our findings about diabetes, Wang et al. ${ }^{(19)}$ found a positive significant association between DED and incident diabetes. The lack of an association between DED and diabetes in our study needs further investigations. Possible reasons for this finding might be the lower prevalence of diabetes compared with the other cardiovascular risks, inadequate sample size and the cross-sectional design of the study. It must also be kept in mind that in our study type 2 diabetes was determined only by the use of FPG, which clearly underestimates the prevalence. To further explore the association of DED and diabetes, one would need to use the $2 \mathrm{~h}$ post-challenge plasma glucose measure as well. Another explanation might be the quantity and quality of dietary fibre, which plays different roles in glucose metabolism ${ }^{(43)}$. It has previously been shown that considering the quantity of total dietary fibre intake without taking into account its food sources might fail to explain its association with the development of impaired glucose disturbance ${ }^{(43)}$. It was shown previously that consumption of energy-dense diets is associated with overweight and obesity ${ }^{(17,30)}$ - these conditions play an important role in developing diabetes and hypertension.

Our findings must be interpreted in the light of some study strengths and limitations. The strengths include the coverage of a broad range of dietary intakes from different socio-economic categories in Tehran, adjustment for important confounders between DED and cardiometabolic abnormalities, and finally the use of a validated FFQ. Limitations of the current study are the crosssectional design of the study, probable misclassification 
of participants due to the use of semi-quantitative FFQ for dietary data collection and confining the participants to women. It must be kept in mind that our sample is representative of Tehran female teachers only, due to the sampling procedure we used. It cannot be regarded as a representative sample of the whole Iranian women or even Tehrani women.

It is concluded that consumption of energy-dense diets is significantly associated with a high prevalence of dyslipidaemia, at least one and at least two cardiometabolic risk factors among Iranian women. Further investigations on larger samples with prospective designs are warranted.

\section{Acknowledgements}

The data collection phase of the study was supported by a grant (P. 25/47/2337) from the National Nutrition and Food Technology Research Institute of the Islamic Republic of Iran and Shaheed Beheshti University of Medical Sciences, Tehran, Iran. Financial support for the conception, design, data analysis and manuscript drafting came from the School of Public Health, Isfahan University of Medical Sciences, Isfahan, Iran. A.E., H.K.B. and L.A. have no personal or financial conflicts of interest. A.E., H.K.B. and L.A. participated in the collection of data, conception and design, statistical analysis and data interpretation, manuscript drafting and approval of the final manuscript for submission. The authors would like to express their grateful appreciation to the Research Council of the Food Security Research Center, Isfahan University of Medical Sciences, for their support.

\section{References}

1. Amuna P \& Zotor FB (2008) Epidemiological and nutrition transition in developing countries: impact on human health and development. Proc Nutr Soc 67, 82-90.

2. Popkin BM \& Gordon-Larsen P (2004) The nutrition transition: worldwide obesity dynamics and their determinants. Int J Obes Relat Metab Disord 28, Suppl. 3, S2-S9.

3. Yusuf S, Reddy S, Ounpuu S et al. (2001) Global burden of cardiovascular diseases: Part II: variations in cardiovascular disease by specific ethnic groups and geographic regions and prevention strategies. Circulation 104, 2855-2864.

4. Naghavi M, Abolhassani F, Pourmalek F et al. (2009) The burden of disease and injury in Iran 2003. Popul Health Metr 7, 9 .

5. Azizi F, Esmaillzadeh A \& Mirmiran P (2004) Obesity and cardiovascular disease risk factors in Tehran adults: a population-based study. East Mediterr Health J 10, 887-897.

6. Esmaillzadeh A \& Azadbakht L (2008) Food intake patterns may explain the high prevalence of cardiovascular risk factors among Iranian women. J Nutr 138, 1469-1475.

7. Mirmiran P, Esmaillzadeh A \& Azizi F (2004) Detection of cardiovascular risk factors by anthropometric measures in Tehranian adults: receiver operating characteristic (ROC) curve analysis. Eur J Clin Nutr 58, 1110-1118.

8. Yusuf S, Hawken S, Ounpuu S et al. (2004) Effect of potentially modifiable risk factors associated with myocardial infarction in 52 countries (the INTERHEART study): case-control study. Lancet 364, 937-952.
9. Esmaillzadeh A \& Azadbakht L (2008) Major dietary patterns in relation to general obesity and central adiposity among Iranian women. J Nutr 138, 358-363.

10. Iqbal SI, Helge JW \& Heitmann BL (2006) Do energy density and dietary fiber influence subsequent 5-year weight changes in adult men and women? Obesity (Silver Spring) 14, 106-114.

11. Du H, van der A DL, Ginder V et al. (2009) Dietary energy density in relation to subsequent changes of weight and waist circumference in European men and women. PLOS ONE 4, e5339.

12. Howarth NC, Murphy SP, Wilkens LR et al. (2006) Dietary energy density is associated with overweight status among 5 ethnic groups in the Multiethnic Cohort Study. J Nutr 136, 2243-2248.

13. Ello-Martin JA, Ledikwe JH \& Rolls BJ (2005) The influence of food portion size and energy density on energy intake: implications for weight management. Am J Clin Nutr 82, 1 Suppl., 236S-241S.

14. Ledikwe JH, Blanck HM, Kahn LK et al. (2006) Dietary energy density is associated with energy intake and weight status in US adults. Am J Clin Nutr 83, 1362-1368.

15. Savage JS, Marini M \& Birch LL (2008) Dietary energy density predicts women's weight change over 6 y. $A m ~ J$ Clin Nutr 88, 677-684.

16. Bes-Rastrollo M, van Dam RM, Martinez-Gonzalez MA et al. (2008) Prospective study of dietary energy density and weight gain in women. Am J Clin Nutr 88, 769-777.

17. Ello-Martin JA, Roe LS, Ledikwe JH et al. (2007) Dietary energy density in the treatment of obesity: a year-long trial comparing 2 weight-loss diets. Am J Clin Nutr 85, $1465-1477$.

18. Saquib N, Natarajan L, Rock CL et al. (2008) The impact of a long-term reduction in dietary energy density on body weight within a randomized diet trial. Nutr Cancer 60, 31-38.

19. Wang J, Luben R, Khaw KT et al. (2008) Dietary energy density predicts the risk of incident type 2 diabetes: the European Prospective Investigation of Cancer (EPIC)Norfolk Study. Diabetes Care 31, 2120-2125.

20. Mendoza JA, Drewnowski A \& Christakis DA (2007) Dietary energy density is associated with obesity and the metabolic syndrome in US adults. Diabetes Care 30, 974-979.

21. Murakami K, Sasaki S, Takahashi Y et al. (2007) Dietary energy density is associated with body mass index and waist circumference, but not with other metabolic risk factors, in free-living young Japanese women. Nutrition 23, 798-806.

22. Willett WC (1998) Nutritional Epidemiology, 2nd ed. New York: Oxford University Press.

23. Esmaillzadeh A \& Azadbakht L (2008) Consumption of hydrogenated versus non-hydrogenated vegetable oils and risk of insulin resistance and the metabolic syndrome among Iranian adult women. Diabetes Care 31, 223-226.

24. Azadbakht L \& Esmaillzadeh A (2009) Red meat intake is associated with metabolic syndrome and the plasma C-reactive protein concentration in women. J Nutr 139, 335-339.

25. Esmaillzadeh A \& Azadbakht L (2008) Home use of vegetable oils, markers of systemic inflammation, and endothelial dysfunction among women. Am J Clin Nutr $\mathbf{8 8}$, 913-921.

26. Esmaillzadeh A, Kimiagar M, Mehrabi Y et al. (2007) Dietary patterns, insulin resistance, and prevalence of the metabolic syndrome in women. Am J Clin Nutr 85, 910-918.

27. Esmaillzadeh A, Kimiagar M, Mehrabi Y et al. (2007) Dietary patterns and markers of systemic inflammation among Iranian women. J Nutr 137, 992-998.

28. Esmaillzadeh A, Kimiagar M, Mehrabi Y et al. (2006) Fruit and vegetable intakes, C-reactive protein, and the metabolic syndrome. Am J Clin Nutr 84, 1489-1497. 
29. Johnson L, Wilks DC, Lindroos AK et al. (2009) Reflections from a systematic review of dietary energy density and weight gain: is the inclusion of drinks valid? Obes Rev $\mathbf{1 0}$, 681-692.

30. Kant AK \& Graubard BI (2005) Energy density of diets reported by American adults: association with food group intake, nutrient intake, and body weight. Int J Obes (Lond) 29, 950-956.

31. Friedwald W, Levy R \& Fredrickson D (1972) Estimation of the concentration of low-density lipoprotein in plasma with use of the preparative ultracentrifuge. Clin Chem 18, 499-502.

32. Craig CL, Marshall AL, Sjostrom M et al. (2003) International physical activity questionnaire: 12-country reliability and validity. Med Sci Sports Exerc 35, 1381-1395.

33. Ainsworth BE, Haskell WL, Whitt MC et al. (2000) Compendium of physical activities: an update of activity codes and MET intensities. Med Sci Sports Exerc 32, 9 Suppl., S498-S504.

34. National Cholesterol Education Program (NCEP) Expert Panel on Detection, Evaluation, and Treatment of High Blood Cholesterol in Adults (Adult Treatment Panel III) (2002) Third report of the National Cholesterol Education Program (NCEP) Expert Panel on Detection, Evaluation, and Treatment of High Blood Cholesterol in Adults. Circulation 106, 3143-3421.

35. Joint National Committee on Prevention, Detection, Evaluation, and Treatment of High Blood Pressure (1997) The sixth report of the Joint National Committee on Prevention,
Detection, Evaluation, and Treatment of High Blood Pressure. Arch Intern Med 157, 2413-2446.

36. Expert Committee on the Diagnosis and Classification of Diabetes Mellitus (2003) Report of the expert committee on the diagnosis and classification of diabetes mellitus. Diabetes Care 26, Suppl. 1, S5-S20.

37. Maldonado G \& Greenland S (1993) Simulation study of confounder-selection strategies. Am J Epidemiol 138, 923-936.

38. Cox DN \& Mela DJ (2000) Determination of energy density of freely selected diets: methodological issues and implications. Int J Obes Relat Metab Disord 24, 49-54.

39. Ledikwe J, Blanck H, Kettel-Kahn L et al. (2005) Dietary energy density determined by eight calculation methods in a nationally representative United States population. $J$ Nutr 135, 273-278

40. Ledikwe JH, Rolls BJ, Smiciklas-Wright H et al. (2007) Reductions in dietary energy density are associated with weight loss in overweight and obese participants in the PREMIER trial. Am J Clin Nutr 85, 1212-1221.

41. Malik VS, Schulze MB \& Hu FB (2006) Intake of sugarsweetened beverages and weight gain: a systematic review. Am J Clin Nutr 84, 274-288.

42. Ledikwe JH, Blanck HM, Khan LK et al. (2006) Low-energydensity diets are associated with high diet quality in adults in the United States. J Am Diet Assoc 106, 1172-1180.

43. Sartorelli DS, Freire RD, Ferreira SR et al. (2005) Dietary fiber and glucose tolerance in Japanese Brazilians. Diabetes Care 28, 2240-2242. 